\title{
Extraperitoneal Spread of Anorectal Abscess: A Case Report and Literature Review
}

\author{
Papadopoulos S. Konstantinos, Dimopoulos Andreas, Kordeni Kleoniki, Filis Dimitrios \\ Deparment of General Surgery, General Hospital of Patras, Patras, Greece
}

Supralevator abscess is a rare form of anorectal disease responsible for very rare but morbid manifestations, one of which is superior spread through fascial planes. We present a rare case of a spreading anorectal abscess in a patient who presented with only diffuse abdominal pain, and we review similar cases in the literature according to anatomical considerations, presentation, diagnostic procedures, and treatment options. We identified 7 previously reported cases of spreading anorectal abscesses. Most abscesses had a horseshoe morphology, and all patients presented or developed abdominal pain. All patients had perianal swelling and pain. Five out of 7 patients were previously mistreated. Only 2 abscesses spread through both the pre- and retroperitoneal planes. Abdominal pain is a dominant feature of extraperitoneal inflammation originating from anorectal abscesses. The absence of perianal signs is rare, and proper inspection of the patient along with the medical history can lead to quicker diagnosis and decisive treatment.

Keywords: Rectal diseases; Abscess; Rectal fistula; Pelvic infection; Surgical drainage; Abdominal pain

\section{INTRODUCTION}

Anorectal abscesses represent one of the most often encountered colorectal diseases in the emergency department (ED) with 70, 000 to 90,000 annual cases in the United States. They occur from blockage or infection of anal crypts and glands due to chronic constipation, inflammatory bowel diseases (IBD; Crohn disease or ulcerative colitis), malignancy, foreign bodies, and sexually transmitted diseases [1]. Parks et al. [2] classified anorectal abscesses into perianal, ischiorectal, intersphincteric, and supralevator [2]. Supralevator abscesses are the least common, occurring in $1 \%$ to $9 \%$ of patients, and they present with nonspecific anal pain and fever while redness or swelling of the buttocks may be absent. Spread across specific anatomic spaces is a very rare complication but may potentially lead to sepsis and death. We report a unique case of supralevator abscess in a patient who presented with only

Received: Nov 27, 2019 - Revised: Jan 6, 2020 • Accepted: Jan 20, 2020 Correspondence to: Papadopoulos S. Konstantinos, M.D.

Deparment of General Surgery, General Hospital of Patras, Tsertidou St. 1, 26332 Patras, Greece

Tel: +30-6975547154, Fax: +30-2610527027

E-mail: dean.papadopoulos66@gmail.com

ORCID: https://orcid.org/https://orcid.org/0000-0003-4171-4096

(C) 2021 The Korean Society of Coloproctology

This is an open-access article distributed under the terms of the Creative Commons Attribution NonCommercial License (https://creativecommons.org/licenses/by-nc/4.0) which permits unrestricted noncommercial use, distribution, and reproduction in any medium, provided the original work is properly cited. diffuse abdominal pain while the abscess expanded massively through both the abdominal and retroperitoneal fascia.

\section{CASE REPORT}

A 57-year-old male presented to the ED with diffuse pain vaguely located at the lower abdomen over the previous 24 hours. He was awake and alert and did not complain of pain in any other site. His past medical history included type 2 diabetes mellitus (DM), gout, myocardial infarction and chronic obstructive pulmonary disease. There was no history of constipation or perianal inflammation. He was a heavy smoker, his body mass index was $45 \mathrm{~kg} /$ $\mathrm{m}^{2}$, and he admitted poor compliance with all of his medications. On admission he was febrile (body temperature, $39.5^{\circ} \mathrm{C}$ ), hypertensive (blood pressure, 160/90 $\mathrm{mmHg}$ ), slightly tachycardic (heart rate, 88 beats/minute), and hyperglycemic (blood glucose, $250 \mathrm{mg} / \mathrm{dL}$ ). Physical examination revealed pain with deep palpation, no rebound tenderness, negative Giordano sign, and no signs of deep vein thrombosis in the extremities. The scrotum and groin were normal. The digital rectal examination was negative for blood but revealed mild nonspecific pain. A plain abdominal radiograph was normal, and laboratory findings consisted of elevated white blood cell count and C-reactive protein (white blood cells, 26,000/ $\mu \mathrm{L}$; neutrophils, $85 \%$; C-reactive protein, $21.58 \mathrm{mg} / \mathrm{L}$ ). Under suspicion of bowel perforation, abdominal contrast-enhanced computed tomography (CT) was performed and revealed 


\section{$\begin{aligned} \text { Annals of } & \text { Extraperitoneal Spread of Anorectal } \\ \text { Coloproctology } & \text { Papadopoulos S. Konstantinos, et al. }\end{aligned}$}

a perirectal fluid-filled cavity and air spreading retroperitoneally and through the abdominal wall fascia (Fig. 1,2) and a perirectal fluid-filled cavity (Fig. 3, 4). In addition, random diverticula were spotted at the left and sigmoid colon. After fluid resuscitation and intravenous antibiotics, the patient was taken to the operating room and underwent a right gluteal incision and intrasphincteric drainage (Fig. 5). A Pezzer tube was placed and used for daily povidone-iodine washes. After 10 days, the patient was afebrile with

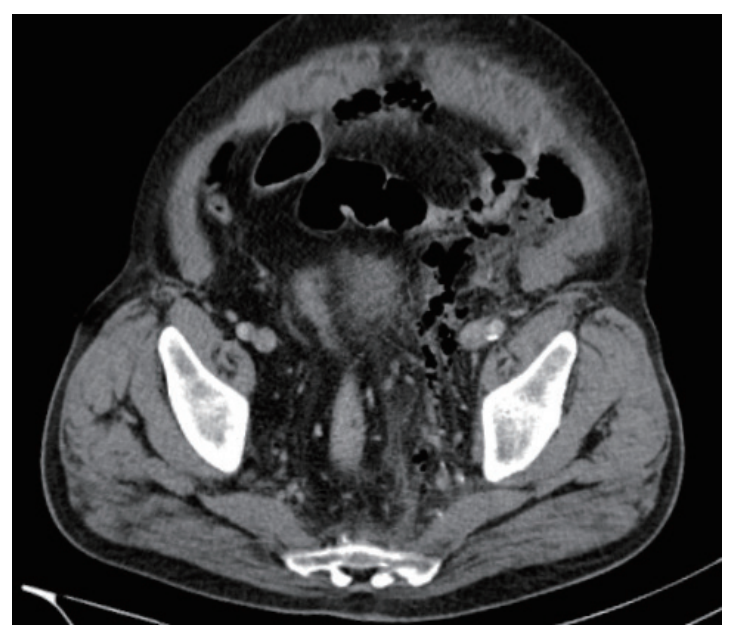

Fig. 1. Axial view showed an emphysematic collection of air at the anterolateral abdominal wall. This image can be easily mistaken for diverticulitis or localized bowel perforation, but with close inspection, the air is spotted extraperitonealy.

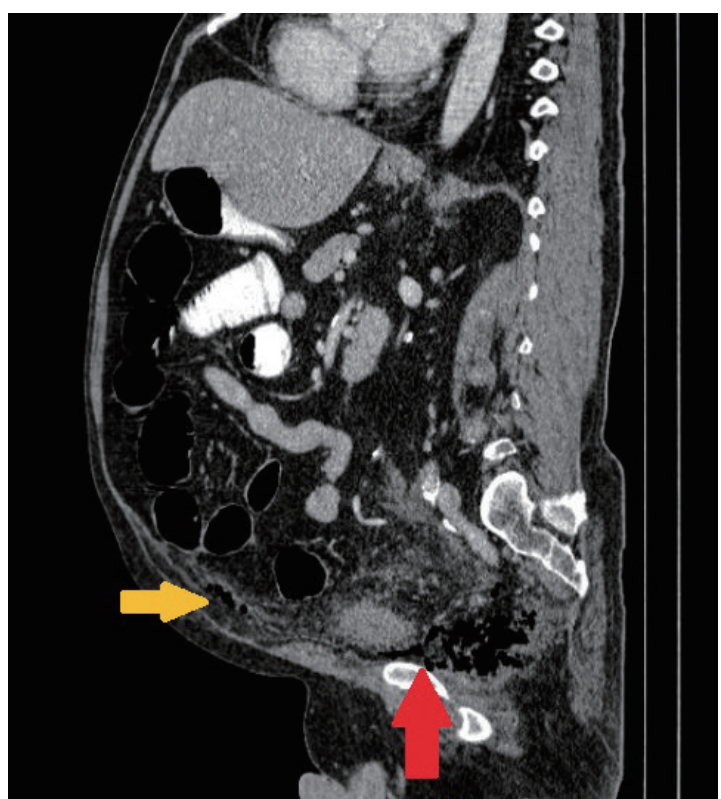

Fig. 2. Sagittal view showed the migration from the supralevator plane (red arrow) preperitoneally (yellow arrow). normal lab values, and he was discharged from the hospital. The patient had no signs of recurrence during a 6-month follow-up period.

Written informed consent for publication of the clinical images was obtained from the patient.

\section{DISCUSSION}

Deep spreading perianal abscesses represent one of the most morbid entities in anorectal disease. Their rarity and insidious clinical manifestations can lead to delayed diagnosis, severe sep-

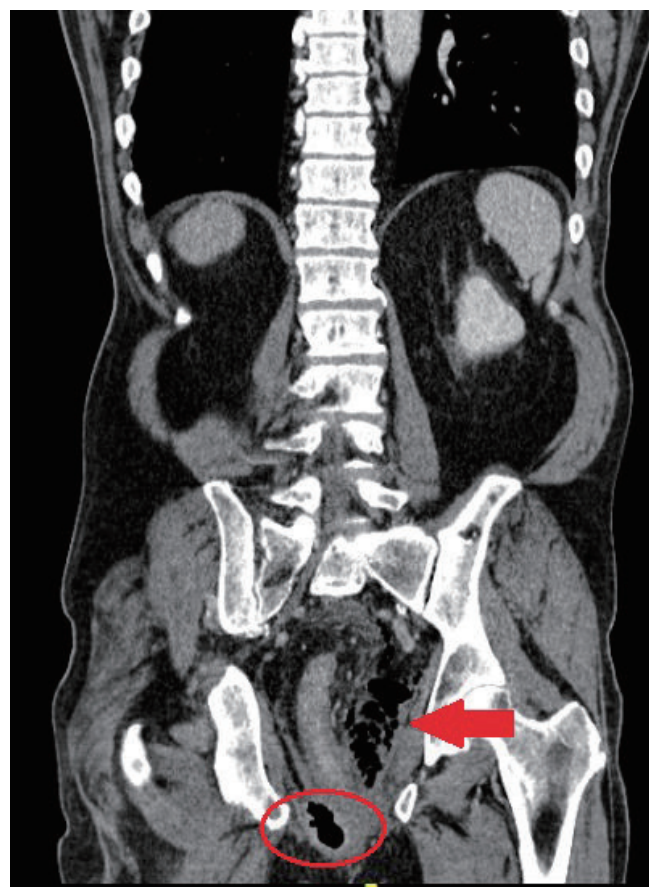

Fig. 3. Frontal view of the abscess cavity on the right side (circle) with contralateral extension to the left suprasphincteric space (arrow).

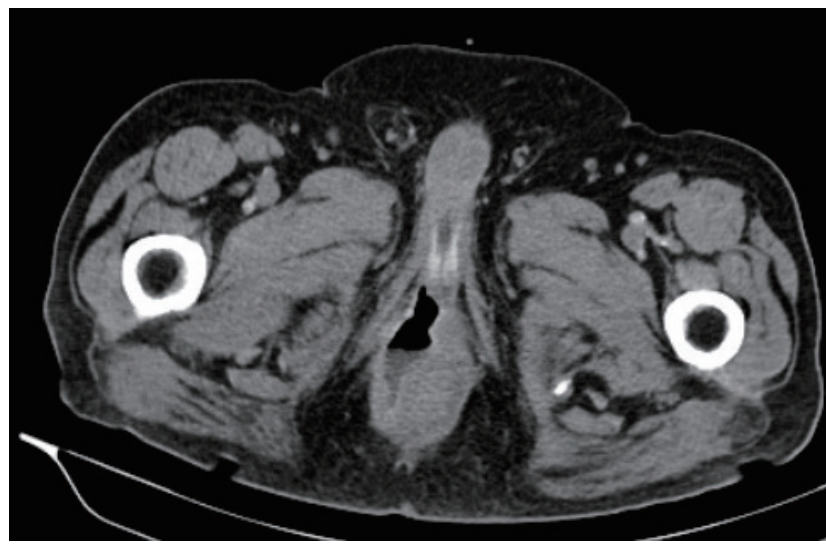

Fig. 4. Fluid-filled cavity with air in axial view. 
sis, and death. Comorbidities, such as IBD, heart disease, obesity, or DM, raise the risk of complications [3], and the absence of typical signs along with abdominal pain can confuse the physician in search of abdominal pathology.

Retroperitoneal abscesses usually originate from the genitourinary tract, may be idiopathic or postoperative, and infrequently involve other organs like the colon, duodenum, and pancreas. Traditionally, lumbar incision was proposed for treatment, with the transperitoneal approach being the least successful, in an overall mortality background of $26 \%$ highly associated with critically ill patients and delayed diagnoses [4].

Anatomically, the supralevator space is a pelvic compartment above the levator ani muscle that communicates anteriorly with the space of Retzius, bilaterally with the retroinguinal spaces, and posteriorly with the retroperitoneum. The puborectalis muscle acts as a barrier to expanding abscesses. However, abscesses can rarely appear above or inside the muscle, usually along with a fistula, allowing the spread of inflammation through both anterior and posterior extraperitoneal abdominal compartments.

The therapeutic plan is vast, and many approaches have been proposed. Percutaneous intersphincteric drainage for limited supralevator abscesses is more difficult, and even fluoroscopic monitoring of the abscess often has inadequate results and might prolong sepsis [5]. The gold standard has always been open drainage and debridement. Fistulotomy can be achieved in one stage with drainage, but there is still some controversy in suprasphincteric abscesses [6]. In abscesses with massive pre- or retroperitoneal extension, access to the peritoneal cavity must be avoided due to

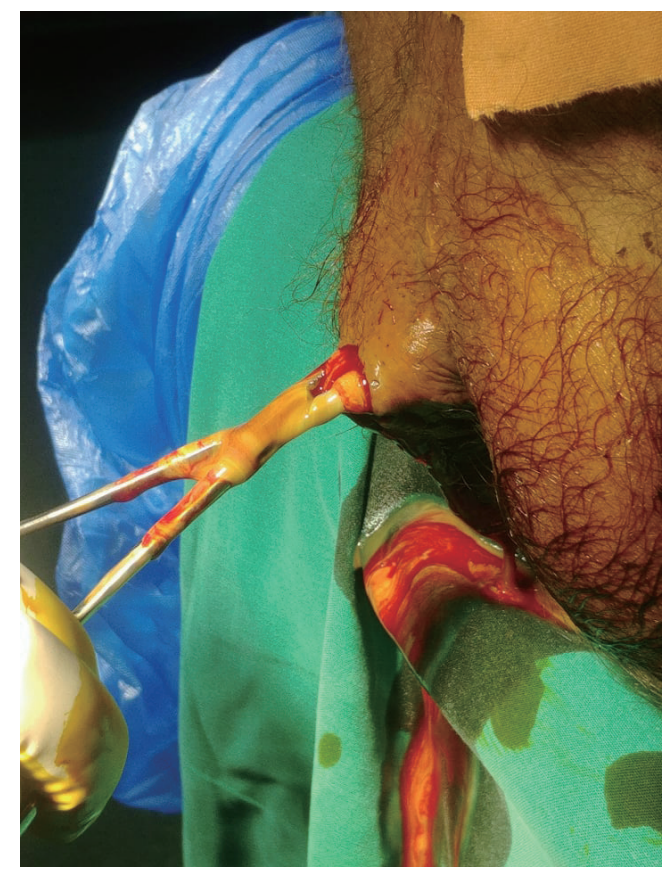

Fig. 5. Drainage of the abscess.

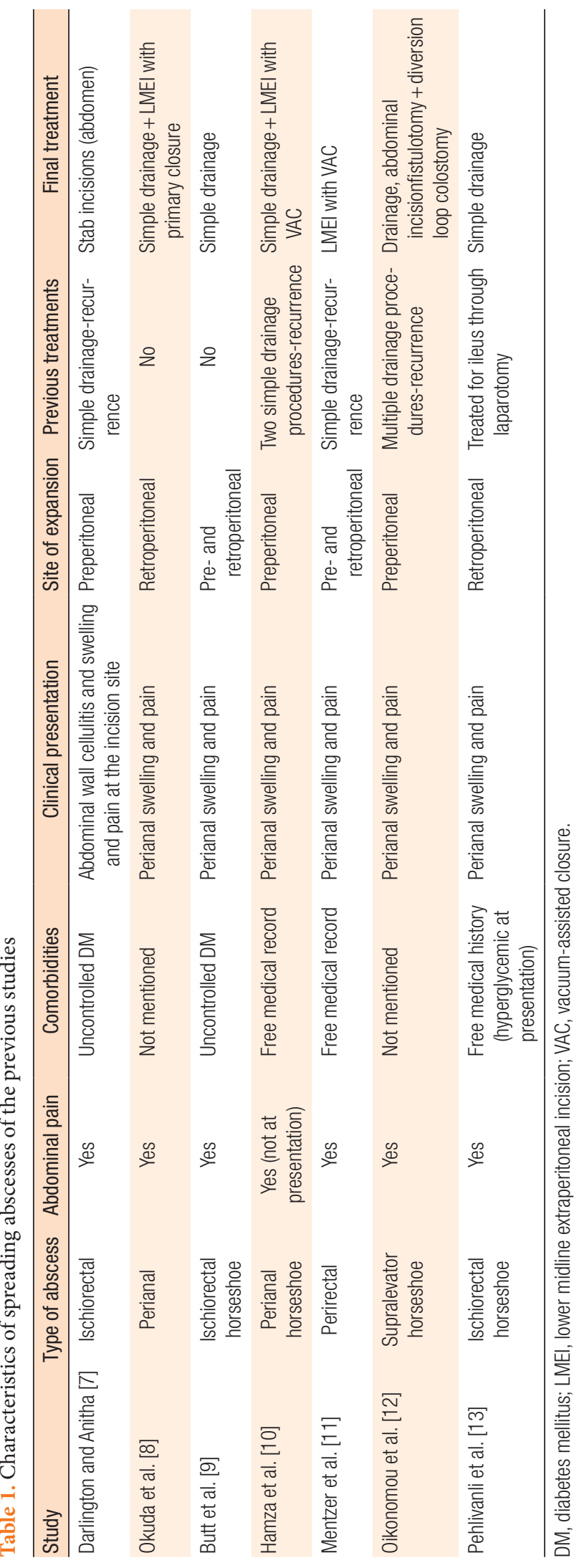


the high risk of contamination and secondary peritonitis. Optimal treatments have been proposed by different authors, including abdominal stab-like incisions [7] or extraperitoneal drainage with lower midline abdominal incision with excellent outcomes $[8,9]$. The wound can be closed primarily with drains or with vacuum assistance devices $[10,11]$. Among the literature, a case of ischiorectal horseshoe abscess with anterolateral extraperitoneal spread was treated with open drainage, canalization through the ischioanal fossa through an abdominal incision, and diversion loop colostomy while performing fistulotomy, all in the same operation [12]. On the contrary, a complicated case of expanding abscess causing ileus was treated successfully with simple drainage after a rather difficult diagnosis [13].

We identified 7 previous cases of anorectal abscesses that fulfilled our criteria of expansion through imaging and clinical presentation (Table 1). To our knowledge, these are all the cases that have been reported. Although there was not a specific type of predominant abscess, 4 out of 7 had a horseshoe morphology. Six patients had abdominal pain at presentation, while the 7th developed abdominal pain with abscess expansion during hospitalization. We conclude that abdominal pain is indicative of an extraperitoneal spreading abscess and a strong indication for further diagnostic studies and imaging, especially in the presence of a previously discovered anorectal abscess. Five patients were either insufficiently treated or misdiagnosed (extension of the primary abscess or the condition itself), and they required a secondary intervention as their conditions worsened. Only 2 out of 7 patients were reported to have uncontrolled DM, but most of the authors did not investigate or did not mention basic medical history elements. All patients survived. We strongly consider early recurrences, after incision, as a major risk factor for abscess expansion, especially if the patient bears comorbidities, such as DM. Lastly, the length of hospital stay ranged from 5 days to 1.5 months with a median of 18 days, although the hospitalization period was not thoroughly analyzed because patients were treated with multiple sessions and sometimes by different surgeons and institutions.

Despite their rarity, extended anorectal abscesses should be considered in differential diagnosis for a septic patient with multiple comorbidities. Immediate resuscitation, CT imaging, and definite intervention are necessary for proper diagnosis and treatment. Expansion of inflammation is unpredictable and can occur in various anatomic planes. Keeping in mind the various manifestations of the disease and its potential spread, physicians could avoid delayed diagnosis or unnecessary laparotomy. Finally, abdominal pain strongly indicates spreading fasciitis, and more aggressive treatment is often needed to ensure a good prognosis for this potentially lethal condition.

\section{CONFLICT OF INTEREST}

No potential conflict of interest relevant to this article was reported.

\section{ACKNOWLEDGMENTS}

We would like to show our gratitude to the Director of Surgery, Spyros Rathosis M.D., Ph.D., for his permission and invaluable guidance about the case.

\section{REFERENCES}

1. Abcarian H. Anorectal infection: abscess-fistula. Clin Colon Rectal Surg 2011;24:14-21.

2. Parks AG, Gordon PH, Hardcastle JD. A classification of fistulain-ano. Br J Surg 1976;63:1-12.

3. Prasad ML, Read DR, Abcarian H. Supralevator abscess: diagnosis and treatment. Dis Colon Rectum 1981;24:456-61.

4. Crepps JT, Welch JP, Orlando R 3rd. Management and outcome of retroperitoneal abscesses. Ann Surg 1987;205:276-81.

5. Sanyal S, Khan F, Ramachandra P. Successful management of a recurrent supralevator abscess: a case report. Case Rep Surg 2012; 2012:871639.

6. Oliver I, Lacueva FJ, Pérez Vicente F, Arroyo A, Ferrer R, Cansado $\mathrm{P}$, et al. Randomized clinical trial comparing simple drainage of anorectal abscess with and without fistula track treatment. Int J Colorectal Dis 2003;18:107-10.

7. Darlington CD, Anitha GF. A rare case of ischiorectal abscess presenting with extensive abdominal wall abscess. Int Surg J 2016; 3:963-4.

8. Okuda K, Oshima Y, Saito K, Uesaka T, Terasaki Y, Kasai H, et al. Midline extraperitoneal approach for bilateral widespread retroperitoneal abscess originating from anorectal infection. Int J Surg Case Rep 2016;19:4-7.

9. Butt UI, Bhatti S, Wadood A, Rehman UA, Changazi SH, Malik K, et al. A case report of pneumo-retro-peritoneum: an unusual presentation of ischio-rectal abscess. Ann Med Surg (Lond) 2017;20: 66-8.

10. Hamza E, Saeed MF, Salem A, Mazin I. Extraperitoneal abscess originating from an ischorectal abscess. BMJ Case Rep 2017;2017: bcr2016218229.

11. Mentzer CJ, Yon JR, King R, Warren JA. Complex perirectal abscess extending to the preperitoneum and space of retzius. GHS Proc 2016;1:49-51.

12. Oikonomou C, Alepas P, Gavriil S, Kalliouris D, Manesis K, Bouboulis $\mathrm{P}$, et al. A rare case of posterior horseshoe abscess extending to anterolateral extraperitoneal compartment: anatomical and technical considerations. Ann Coloproctol 2019;35:216-20.

13. Pehlivanli F, Aydin O, Karaca G, Aydin G, Erden Daphan Ç. An extremely rare complication of widespread retroperitoneal abscess originating from anorectal horseshoe abscess. Bull Emerg Trauma 2019;7:72-5. 